\title{
Of margins and men-do surgical margins matter?
}

Two recent papers draw attention to the importance of surgical margins in men undergoing radical prostatectomy.

In a paper published in the Journal of Urology, researchers from Johns Hopkins discussed the importance of recording Gleason score at the surgical margin, as well as whether it was positive for tumour histology. 4,082 patients who have undergone radical prostatectomy since 2010 were included in the study; such information has been routinely collected at the institution for the past 5 years.

In patients with positive margins, $22 \%$ experienced biochemical recurrence, $3 \%$ metastases, and 30\% required adjuvant radiation. Interestingly, a shorter positive margin was associated with lower Gleason score at the margin, and a lower grade margin was independently associated with a reduced risk of recurrence (HR 0.50; OR 0.25-0.97). "Essentially, among patients with prostate cancer undergoing radical prostatectomy who have a pathologic positive margin, the biology of the disease at the margin might be significant," explains
Max Kates, lead author of the study. "The concept that a lower pathologic grade at the margin portends better prognosis is potentially useful clinically in counseling patients postoperatively."

A second article, published in Urologic Oncology, describes the importance of "close" but negative surgical margins in prognosticating biochemical recurrence after radical prostatectomy. Using the Columbia University Urologic Oncology Database, Whalen and colleagues retrospecitvely reviewed pathology reports of men who had undergone radical prostatectomy between 2005 and 2011. Surgical margins were classified using a cut-off value of $<1 \mathrm{~mm}$ for close margins. Patients were monitored postoperatively with routine clinic appointments, serial serum PSA tests, and imaging, as required; the primary end point was the presence of biochemical recurrence. After a median 20.5 months follow-up period, $8.2 \%, 16.7 \%$ and $24.6 \%$ of patients had experienced biochemical recurrence in the negative, close and positive margin groups, respectively. "While patients with close margins had pathologic characteristics more similar to the patients with negative margins, their rates of biochemical failure at 3 years were more similar to patients with positive margins," corresponding author Michael Whalen told Nature Reviews Urology. "Thus, the influence of the surgical margin relies on more nuanced information than 'positive' or 'negative'. Clearly, this association needs to be studied further on a larger scale ... it is our recommendation that the margin distance be explicitly mentioned in surgical pathology reports along with primary Gleason score”.

Annette Fenner

Original articles Whalen, M. J. et al. Close surgical margins after radical prostatectomy mimic biochemical recurrence rates of positive margins. Urol. Oncol. doi:10.1016/j.urolonc.2015.07.005 | Kates, M. et al. Importance of reporting the Gleason score at the positive surgical margin site: an analysis of 4,082 consecutive radical prostatectomy cases. J. Urol. doi:10.1016/ j.juro.2015.08.002 